\title{
Inflammatory Breast Cancer in Tunisia from 2005 to 2010: Epidemiologic and Anatomoclinical Transitions from Published Data
}

\author{
N Mejri ${ }^{1 *}$, H Boussen ${ }^{1}$, S Labidi ${ }^{1}$, H Bouzaiene ${ }^{2}$, M Afrit ${ }^{1}$, F Benna ${ }^{2}$, K Rahal ${ }^{3}$
}

\begin{abstract}
Aim: To report epidemiologic and anatomoclinical transitions of inflammatory breast cancer (IBC) in Tunisia. Materials and Methods: Data including clinico-pathological data for208 cases of T4d or PEV 3 non-metastatic breast cancer diagnosed between 2005 and 2010 were collected from patient records. Chi 2 and $Z$ tests were used to compare variables with two Tunisian historical series and a series about Arab-American patients. Results: Thirty three percent of our patients had their first child before 23 years of age and $56 \%$ had their menarche before 12 years, $75 \%$ never receiving oral contraception. Obesity was observed in $42 \%$ of women and IBC occurred during pregnancy in $13 \%$ of cases. Tumor grade was II-III in $90 \%$ of cases, HR was negative in $52 \%$, HER2 was over expressed in $31 \%$ and invasion of more than 3 axillary nodes occurred in $18 \%$ of patients. We observed a pCR rate of $19 \%$ after neoadjuvant treatment (anthracyline-taxane used in 79\%, trastuzumab in $27 \%$ ). Compared to historical Tunisian series (since 1996), IBC epidemiology remained stable in terms of median age, menopausal status and obesity. However we observed a significant decrease in median clinical tumor size and number of positive axillary lymph nodes. Comparison to IBC in Arab-Americans showed a significant difference in terms of median age, menopausal status, positivity of hormonal receptors and educational level. Conclusions: Our assessment of epidemiologic transition showed a reduction of clinco-pathological stage of IBC, keeping the same characteristics as compared to Tunisian historical series over a period of 14 years. Features seem to be different in Arab-American patients, probably related to migration, "occidentalization" of life style and improvement in socio-economic level
\end{abstract}

Keywords: Inflammatory breast cancer - epidemiology - ethnicity - Tunisia - Arab-Americans

Asian Pac J Cancer Prev, 16 (3), 1277-1280

\section{Introduction}

Inflammatory breast cancer (IBC) is a particular entity within Breast Cancers (BC), characterized by highly aggressive clinical and evolutionary patterns and high incidence in emerging countries (North Africa, Egypt) (Levine and Veneroso, 2008). Therapeutic interest to IBC is mainly explained by the young mean age of patients, the high rate of chemo-resistance and the frequency of distant failures (Dawood et al., 2014).

Interest to IBC was marked by several publications devoted to epidemiology, histopathology and molecular biology leading to individualization of new molecular targets and therapeutic perspectives. First epidemiologic IBC studies arose from Tunisian American and European teams within an international multicentric collaboration in the seventies. Those studies have been greatly hampered by use of inconsistent diagnostic criteria. In Tunisia a high incidence (50\%) was reported since the opening of the Salah Azaiz Institute in 1969 (Mourali et al., 1980).
Since that period a marked epidemiologic transition of IBC in Tunisia was observed in the series collected until 2000 showing a steady decline in IBC cases (Boussen et al., 2010). This decrease appears to be explained by improvement of socio-economic level (SEL) in our country, with an increase of the growth domestic product (Shahbaz and Lean, 2012) and more stringent and rigorous clinical criteria for IBC diagnosis. Although risk factors for IBC remain unclear, some established risk factors including ethnicity, obesity, socio-economic level and young age may explain differences in epidemiological aspects between North-African, US and European populations (Schlichting et al., 2012). The importance of SEL as a risk factor of IBC is suggested by the rural predominance of this entity in Tunisia and the reported different profile between North African migrants to France and French women living in the same region (Arriagada MG et al., 2005).

The aim of the present retrospective study concerning patients with IBC recently treated from 2005 to 2010 is to 
Table 1. Clinico-pathological Transition of IBC between Historical Series between 1996 and 2010

\begin{tabular}{|c|c|c|c|c|c|}
\hline Period & & $\begin{array}{c}\text { Boussen(A) } \\
(n=118) \\
1996-1999\end{array}$ & $\begin{array}{c}\text { Labidi (B) } \\
\quad(n=100) \\
1994-2000\end{array}$ & $\begin{array}{l}\text { Our serie }(C) \\
\quad(n=208) \\
2005-2010\end{array}$ & $\begin{array}{l}\mathrm{C} \text { vs A } \\
\mathrm{C} v s \mathrm{~B}\end{array}$ \\
\hline \multirow{11}{*}{ Nodal involvement } & Age (years) & 43 & 43 & 45 & ns \\
\hline & Under 45 years $(\%)$ & & 61 & 62 & ns \\
\hline & Premenopause (\%) & 89 & 70 & 61 & $<0.02 \mathrm{~ns}$ \\
\hline & Pregnancy (\%) & NA & 10 & 13 & ns \\
\hline & Obese $\mathrm{BMI}>30(\%)$ & 43 & 42 & 42 & ns \\
\hline & Negative HR (\%) & NA & 67 & 52 & 0.04 \\
\hline & Grade II-III (\%) & 94 & 86 & 90 & ns \\
\hline & Median clinical tumor size & 90 & 53 & 63 & $<0.001$ \\
\hline & 0 & 0 & 8 & 28 & $<0.001$ \\
\hline & $1-3$ & 17 & 83 & 52 & $<0.001$ \\
\hline & $>4$ & 77 & 8 & 18 & $<0.001$ \\
\hline
\end{tabular}

report epidemiologic transition of IBC in Tunisia within a period of 14 years (1996-2010) and to evaluate anatomoclinical and therapeutic results observed. We also try to demonstrate the role of SEP on IBC profile by comparing our population to an Arab-American population published from surveillance, epidemiology and end results registries data base (SEER).

\section{Materials and Methods}

We carried out a retrospective study of histologically confirmed non metastatic IBC defined by rapid onset of edema and erythema with or without underlying palpable mass. Clinical classification considered T4d stage according to AJCC classification (6th edition) or PEV3 according to French IGR classification. All patients were treated with curative intent in Salah Azaiez Institute (ISA) and University Hospital Abderrahman Mami (UHAM) in Tunis, Tunisia between 2005 and 2010. Clinico-pathological information was collected. Baseline information included age, menopausal status, BMI, SEL, pregnancy period, hormonal receptors, HER2 status, nodal involvement and grade, therapeutic modalities and results. We compared our results to two historical Tunisian series concerning patients treated before 2000; the first serie published by Labidi et al including patients treated between 1994 and 2000 [8], the second published by Boussen et al including patients treated between 1996 and 1999 [4]. We also compared our results with an Arab-American based population study published by Hirko et al including patients treated between 1988 and 2008 from SEER database [9]. This study examined the occurrence of IBC among Arab Americans in California, Detroit and New Jersey which is the largest Arab American population sample published in the literature. Differences in the proportion IBC characteristics were evaluated using chi-square tests for categorical variables and $\mathrm{Z}$ test for continuous variables. Statistical significance was reached if $\mathrm{p}<0.05$.

\section{Results}

We collected 208 patients treated between 2005-2010 at ISA and UHAM. Mean age was 45 years (range 2572) with $62 \%$ younger than 35 years. We observed $61 \%$
Table 2. Differences and Similarities between Tunisian and Arab-American Population

\begin{tabular}{lccc}
\hline & Our serie $(\mathrm{n}=208)$ & SEER $(\mathrm{n}=94)$ & $\mathrm{P}$ \\
\hline Period & $2005-2010$ & $1988-2008$ & \\
Ethnicity & Arab-Tunisia & Arab-Americans \\
Age & 45 & 58 & $<0.001$ \\
Premenopause & $63 \%$ & $26 \%$ & $<0.001$ \\
Educational level & & & \\
$\quad$ Low+Middle & $77 \%$ & $59.30 \%$ & $<0.001$ \\
$\quad$ High & $19 \%$ & $38.30 \%$ & $<0.001$ \\
Hormonal receptors negative & $52 \%$ & $45.20 \%$ & 0.05 \\
HER2 overexpressed & $31 \%$ & $29.20 \%$ & 0.28 \\
\hline
\end{tabular}

of patients in premenopausal and $13 \%$ diagnosed during pregnancy. Obesity defined by $\mathrm{BMI} \geq 30$ was seen in $42 \%$ of cases. Seventy seven percent of our population had low and middle educational level.

Age under 23 years at first birth was observed in 33\% of cases, age under 12 years at menarche was seen in $56 \%$ of women. Sixty three percent of women had more than one child and $75 \%$ never received oral contraception.

Mean clinical tumor size at diagnosis was $63 \mathrm{~mm}$. Infiltrating lobular carcinoma was observed in $3 \%$ of cases. Most patients (52\%) had more than 3 invaded axillary lymph nodes and $28 \%$ has no involved lymph nodes. Hormonal receptors were negative in $52 \%$ of cases and HER 2 was over expressed in $31 \%$ of cases. Grade II-III was seen in $90 \%$ of patients.

All patients received neoadjuvant chemotherapy: $79 \%$ based on Anthracylines and Taxanes, 2\% using Taxanes alone and 19\% using Anthracyclines alone. Only 27\% of HER2 over expressing patients received trastuzumab. Pathological complete response was obtained in $19 \%$ of patients.

When compared our results with the available data of historical series of Boussen et al and Labidi et al. (Table 1), no statistically significant difference in terms of age, proportion of young women ( $<35$ years), diagnosis during pregnancy, obesity and tumor grade was observed. However less premenopausal women (61\% vs 89\%), smaller clinical tumor size at diagnosis (63mm vs $90 \mathrm{~mm})$, lower proportion of hormonal receptors negative cases (52\% vs 67\%) and less nodal involvement was observed (52\% vs $77 \%$ ) with a significant $\mathrm{p}$ value. 
We compared also our results with available data about Arab-American IBC cases from the SEER database (Table 2), our patients were significantly younger (45 $v s 58$ years, $\mathrm{p}<0.001)$, more frequently premenopausal (63\% vs 26\%) and had lower education level (19\% vs $38 \%$ ). However similar immunohistochemical features in terms of hormonal receptors (52\% vs 45\%) and HER2 over expression (29.2\% vs 31\%) was observed between both populations.

\section{Discussion}

Our present study and the comparison to previous IBC Tunisian cases, confirmed some epidemiologic transition of this particular disease with an increase of SEL and a decrease of tumor size. The incidence of IBC is high in Arab countries (Tunisia 5\%, Morocco 11\%, Egypt 6\%) and it was also found higher in Arab-American $(\mathrm{OR}=1.5,95 \%, \mathrm{CI}=1.2,1.9)$ compared to other American ethnicities (Hispanics, Indians, Alaskans) in the SEER series (Chouchane et al., 2013; Corbex et al., 2014). Some epidemiologic features of Tunisian IBC remain stable; occurring in young/very young patients despite a significant increase of socio-economica and educational level. The significant decrease in tumor size at diagnosis from $90 \mathrm{~mm}$ to $53 \mathrm{~mm}$ in the recent series is mainly due to the major effort of publications by Najib Mourali team since the opening of ISA in 1970 until the nineties reducing. This epidemiologic transition is also probably related to the increase of socio-economic level in our country. Schilichting et al. demonstrated that living in a high poverty country, low educational level and low SEL is significantly associated with IBC (Schlichting et al., 2012). This relationship was not validated for overall breast cancer which was associated with urban residency with high SEL in several studies (Robert SA et al., 2004). Young age remains the most stable characteristic of IBC in Tunisia, currently perceived by many as a disease of younger women. Mean age of IBC reported in Tunisian series was 43-45 year-old, remaining steady within a period of 14 years. Similar median age was reported in an Indian series (Gogia et al., 2014). A significant difference was seen in comparison with Arab American. A recent SEER data base study published by Schinkel et al. in 2014 reported that IBC, disproportionately affects older women (Schinkel et al., 2014). The mean age at IBC diagnosis has been estimated at 55 years (per the M. D. Anderson IBC Registry) and at 58.7 years (per the SEER 13 Registry, 1992-2002) and it rises sharply with age until it plateaus after age 65 [Goldner B, et al., 2014). Younger age at menarche was reported to be a risk factor of IBC since the first Tunisian series published by Mourali et al. in 1980 (Mourali et al., 1980) until Boussen et al., 2010 (Boussen H et al., 2010) as well as Leuvin et al. in 2008 publications (Levine et al., 2008). Early age at first birth, pregnancy and breast feeding exceeding 24 months may also, be risk factors for aggressive breast cancer in general and IBC in particular. In a series of 215 breast cancer patients, reported by Veneroso et al. women who had their first child before age 20 years had approximately 3.2 fold increased odds of having a high-grade breast cancer
(OR, 3.20; 95\% CI, 1.20-8.49) (Veneroso et al., 2008). In our series, $33 \%$ of women had their first child before the age of 23. In Mourali's series, pregnancy was associated with an incidence of $79 \%$ of rapidly progressing breast cancer versus $57 \%$ for non rapidly progressing breast cancer $(p=0.01)$. In our series only $13 \%$ of IBC cases were diagnosed during pregnancy. The role of menopausal status is not yet clear. In the Arab-Americans, only $26.6 \%$ of women were diagnosed with IBC in the premenopausal years, which was the lowest proportion compared to other ethnicities. However premenopausal women had a significantly improved mean survival as compared to postmenopausal women $(96.4$ vs $59.2, \mathrm{p}<0.0001)$ this finding makes the consideration of menopausal status as risk/prognostic factor controversial (Schlichting et al., 2012; Amiri-Kordestani 2013). Obesity is now a validated risk factor of overall breast cancer and IBC in particular. The results of Chairer et al. showed a striking fourfold increase of IBC in obese women compared to normal weight women. Chang et al. also found that high BMI was significantlly associated with increased risk of IBC regardless of menopausal status (Chang et al., 1998). In our series and also in historical Tunisian series obesity was associated to IBC in almost $50 \%$ of cases (Robertson et al., 2010; Amiri-Kordestani 2013).

The most important transition of IBC management in Tunisia was the introduction of Taxanes and Trastuzumab in the neoadjuvant setting. This improvement in management translated into a significant decrease in nodal involvement, reduction in massive nodal involvement rate $(\geq 4 \mathrm{~N}+)$ from $77 \%$ in the 90 's to $18 \%$ in our series. International expert panel on IBC consensus agreed to recommend that all women with IBC should receive a primary systemic regimen consisting of an anthracycline and taxanes. The panel also strongly recommends the administration of trastuzumab among women with HER2positive disease (Rehman et al., 2012).

Some weakness should be noted in our study. The comparative Arab-American population is heterogeneous with luck of data on origin country and date of immigration, which could be responsible for differences with our population. Some compared factors are not standardized between the series which does not allow accurate conclusions. We also considered educational level as proxy for SEL, assuming that patients with low educational level are more likely to have a low SEL; this assumption is not always validated especially in Tunisian population. Additionally, we luck data about reproductive risk factors (menarche, pregnancy), pathology features (grade, IHC technique) which may be directly responsible for disparities between Tunisian and Arab-American IBC patients.

In conclusion, IBC frequency in Tunisia is presently lower (5\%-7\%) than in the 1970s. This incidence/ frequency decrease could be explained by the effect of early diagnosis, awareness campaigns, and improvement of the SEL in Tunisia. This probable epidemiologic transition shows as a reduction of clinco-pathological stage of IBC, keeping the same characteristics as compared to Tunisian historical series over a period of 14 years. Features seem to be different in Arab-American 


\section{N Mejri et al}

patients probably related to migration, "occidentalization" of life style and improvement in socio-economic level. IBC epidemiologic differences between populations suggests that its pathogenesis involves factors such as genetics, age of menarche, body mass index, diet, and other lifestyle aspects..

\section{References}

Amiri-Kordestani L, Kamangar F, Zujewski JA (2013). Inflammatory breast cancer: yet another risk of the obesity epidemic? J Natl Cancer Inst, 105, 1340-2.

Boussen H, Bouzaiene H, Ben Hassouna, et al (2010). Inflammatory breast cancer in Tunisia. Cancer, 116 (S11), 2730-35.

Chang S, Buzdar AU, Hursting SD (1998). Inflammatory breast carcinoma incidence and survival. Cancer, 82, 2366-72.

Chouchane L, Boussen H, Sastry KS (2013). Breast cancer in Arab populations: molecular characteristics and disease management implications. Lancet Oncology, 14, 417-424.

Corbex M, Bouzbid S, Boffetta P (2014). Features of breast cancer in developing countries, examples from North-Africa. Eur J Cancer, 50, 1808-18.

Dawood S, Lei X, Dent R (2014). Survival of women with inflammatory breast cancer: a large population-based study. Ann Oncol, 25, 1143-51.

Gogia A1, Raina V, Deo SV, et al (2014) Inflammatory breast cancer: a sinqory breast cancer in women, 1992-2009, United States. Ann Surg Oncol, 21, 1267-270

Hance KW, Anderson WF, Devesa SS, et al (2005). Trends in inflammatory breast carcinoma incidence and survival: the surveillance, epidemiology, and end results program at the National Cancer Institute. J Natl Cancer Inst, 97, 966-75.

Hirko KA, Soliman AS, Banerjee M, et al (2013). Characterizing inflammatory breast cancer among Arab Americans in the California, Detroit and New Jersey Surveillance, Epidemiology and End Results (SEER) registries (19882008). SpringerPlus, 2, 1-8.

Labidi SI, Mrad K, Mezlini A, et al (2008). Inflammatory breast cancer in Tunisia in the era of multimodality therapy. Ann Oncol, 19, 473-80.

Lè MG, Arriagada R, Contesso G, et al (2005). Dermal lymphatic emboli in inflammatory and noninflammatory breast cancer: a French-Tunisian joint study in 337 patients. Clin Breast Cancer, 6, 439-45

Levine PH, Veneroso C (2008). The epidemiology of inflammatory breast cancer. Seminars in oncology, 35, 11-16

Mourali N, Muenz LR, Tabbane F, et al (1980). Epidemiologic features of rapidly progressing breast cancer in Tunisia. Cancer, 46, 2741-6.

Natori A, Hayashi N, Soejima K, et al (2013). A comparison of epidemiology, biology, and prognosis of inflammatory breast cancer in Japanese and US populations. Clin Breast Cancer, 13, 460-4.

Rehman S, Reddy CA, Tendulkar RD (2012). Modern outcomes of inflammatory breast cancer. Int J Radiat Oncol Biol Phys, 84, 619-24.

Robert SA, Strombom I, Trentham-Dietz A, et al (2014). Socioeconomic risk factors for breast cancer: distinguishing individual- and community-level effects. Epidemiology, 15, 442-50.

Robertson FM, Bondy M, Yang W, et al (2010). Inflammatory breast cancer: the disease, the biology, the treatment. $C A: A$ Cancer J Clin, 60, 351-75.

Schairer C, Li Y, Frawley P, et al (2013). Risk factors for inflammatory breast cancer and other invasive breast cancers.
J Natl Cancer Inst, 105, 1373-84.

Schlichting J, Solima AS, Schairer C, et al (2012). Association of inflammatory and noninflammatory breast cancer with socioeconomic characteristics in the Surveillance, Epidemiology, and End Results Database, 2000-2007. Cancer Epidemiology Biomarkers \& Prevention, 21, 155-65.

Schlichting JA, Soliman AS, Schairer C, et al (2012). Inflammatory and non-inflammatory breast cancer survival by socioeconomic position in the Surveillance, Epidemiology, and End Results database, 1990-2008. Breast Cancer Res Treat 134, 1257-68.

Schinkel JK, Zahm S H, Jatoi I, et al (2014). Racial/ethnic differences in breast cancer survival by inflammatory status and hormonal receptor status: an analysis of the surveillance, epidemiology, and end results data. Cancer Causes Control, $1-10$.

Shahbaz M, Lean HH (2012). Does financial development increase energy consumption? The role of industrialization and urbanization in Tunisia. Energy Policy, 40, 473-9.

Veneroso C, Siegel R, Levine PH (2008). Early age at first childbirth associated with advanced tumor grade in breast cancer. Cancer Detect Prev, 32, 215-223 